\begin{tabular}{|c|l|}
\hline Title & Excitation Wavelength Dependent Three W ave Mixing at a CO-Covered Platinum Electrode \\
\hline Author(s) & Y agi, Ichizo; Nakabay ashi, Seiichiro; Uosaki, Kohei \\
\hline Citation & $\begin{array}{l}\text { Journal of Physical Chemistry B, 101/38), 7414.7421 } \\
\text { https://doi.org/10.1021/jp964010r }\end{array}$ \\
\hline Issue Date & 1997-09-18 \\
\hline Doc URL & http://hdl.handle.net/2115/50247 \\
\hline Type & article \\
\hline File Information & JPCB101-38_7414_7421.pdf \\
\hline
\end{tabular}

Instructions for use 


\title{
Excitation Wavelength Dependent Three-Wave Mixing at a CO-Covered Platinum Electrode
}

\author{
Ichizo Yagi, Seiichiro Nakabayashi, ${ }^{\dagger}$ and Kohei Uosaki* \\ Physical Chemistry Laboratory, Division of Chemistry, Graduate School of Science, Hokkaido University, \\ Sapporo 060, Japan
}

Received: December 6, 1996; In Final Form: June 30, $1997^{\otimes}$

\begin{abstract}
The interfacial electronic structure of a CO-covered polycrystalline platinum electrode has been studied by using the optical second harmonic generation (SHG) and sum frequency generation (SFG) techniques with various excitation wavelengths. Although the nonlinear optical (NLO) signal was enhanced by the absorption of $\mathrm{CO}$ at all excitation wavelengths employed in this study, the potential dependent behaviors of the NLO signal were different between the near-infrared excitation and the visible excitation. The difference was attributed to the different mechanisms for the enhancement of the NLO signal. While the electron transition from the Fermi level of Pt to the unoccupied $2 \pi_{\mathrm{a}}{ }^{*}$ orbital of adsorbed $\mathrm{CO}$ was considered to be coupled with the NLO photon in the case of visible excitation, the dc field induced SHG regime was applied to the result obtained by the near-infrared excitation.
\end{abstract}

\section{Introduction}

The electrochemical oxidation of small organic molecules, e.g., methanol, formaldehyde, and formic acid, at a platinum electrode is a technologically important catalytic process that is utilized in future energy technology, e.g., direct methanol fuel cell. It is well-known that the adsorbed $\mathrm{CO}(\mathrm{CO}(\mathrm{a}))$ which is formed dissociatively from a parent molecule behaves as a poison for the direct oxidation of the parent molecule and other electrochemical reactions. ${ }^{1-12}$ Accordingly, knowledge about the mechanisms of both the absorption and the oxidation of CO(a) is very important from the industrial point of view. The fundamental aspects of electrochemical surface science are concerned with the microscopic understanding of the bonding and the reactivity of absorbates which are influenced by morphological and electronic structures of the interface. CO(a) on Pt is one of the ideal adsorbates in this respect because much information has been obtained by UHV studies. ${ }^{13-18}$ The electrochemical behavior of the $\mathrm{CO} / \mathrm{Pt}$ system has been investigated by using various techniques including infrared reflection absorption spectroscopy (IRAS), ${ }^{6-11,19-27}$ differential electrochemical mass spectrometry (DEMS), ${ }^{10,11}$ electrochemical quartz crystal microbalance (EQCM) ${ }^{28-30}$ and surface-enhanced Raman scattering (SERS). ${ }^{12,31}$ Although these methods are very powerful for the quantitative analysis of the adsorbate and investigation of the oxidation mechanism, they cannot provide direct information about interfacial electronic structure.

Three-wave-mixing processes such as optical secondharmonic generation $(\mathrm{SHG})^{32}$ and optical sum frequency generation $(\mathrm{SFG})^{32}$ have been recognized as novel in situ optical probes of surface electronic and geometric structures. Optical SHG is one of the second-order nonlinear optical effects, and it is the conversion of two photons of frequency $\omega$ into a single photon of frequency $2 \omega$ with laser irradiation. In the electric dipole approximation, even order nonlinear optical effects require noncentrosymmetric media. Thus, SHG cannot be observed in centrosymmetric media such as bulk metals and

* Corresponding author. Fax: +81-11-706-3440. E-mail: uosaki@ pcl.chem.hokudai.ac.jp.

$\dagger$ Present address: Department of Chemistry, Faculty of Science, Saitama University, Urawa 338, Japan.

${ }^{\otimes}$ Abstract published in Advance ACS Abstracts, August 15, 1997. solutions and at interfaces between two centrosymmetric media. The SHG process can occur only within a few atomic layers of both sides of the interface where inversion symmetry is broken. Consequently, the SHG signal is sensitive to changes at the surface and interface, and therefore, the SHG process is a useful probe of various interfacial phenomena. This technique has been applied to various systems including electrochemical systems to probe the dc field ${ }^{33-36}$ and geometric and electronic structure of interfaces ${ }^{37-42}$ and is often used to study the adsorption of molecules, ions, and metal atoms on various interfaces in both resonant and nonresonant conditions. ${ }^{32,40-42}$ The resonant enhancement of the SHG signal is observed when the electronic transition couples energetically with either the input photon or the output $\mathrm{SH}$ photon. The surface electronic structures of semiconductors ${ }^{42-44}$ and metals ${ }^{45-49}$ and molecular electronic transitions at liquid/gas and liquid/liquid interfaces ${ }^{50-52}$ have been investigated by utilizing this effect. The resonant SHG signal should be dominated by interfacial features of the specific chemical species, and therefore, the analysis of the resonant SHG profile should provide a simple picture of interfacial phenomena. On the other hand, nonresonant $\mathrm{SHG}^{53-55}$ is an indirect probe of interfacial phenomena via perturbation and redistribution of the surface electronic structure as a result of absorption and requires a complex analysis of the signal. This approach is most useful to study electron-rich surfaces such as metals and semiconductors.

SFG is inherently the same process as SHG. In SFG, two photons of frequency $\omega_{1}$ and $\omega_{2}$ are converted into a single photon of frequency $\omega_{1}+\omega_{2}$. Actually, SHG can be considered as the special case of SFG when $\omega_{1}=\omega_{2}$. Although the optical SFG technique has been mostly applied to the vibrational spectroscopy at surfaces and interfaces, ${ }^{56-58}$ it has been also used to supplement electronic $\mathrm{SH}$ spectroscopy in a few studies. ${ }^{59-61}$ Since $\mathrm{SH}$ enhancement can take place by resonance of electron transitions at surfaces with either the incident fundamental or generated SH photons, one cannot distinguish which photon contributes to the resonance. This problem should be solved and the resonant energy can be determined using the SFG technique together with the SHG technique. For example, Heinz et al. carried out both SHG measurements with tunable visible light from a dye laser and SFG measurements with 
tunable visible light and fixed near-infrared light to study a $\mathrm{CaF}_{2} /$ $\mathrm{Si}(111)$ interface ${ }^{59}$ and found that the resonant line was observed at the same visible input wavelength. Since the SH wavelength was different from the SF wavelength with the same input visible light, the resonance at the visible input wavelength was confirmed. In addition, SFG can extend the wavelength region to be investigated, although the experimental simplicity of SHG is weakened.

In our previous publications, ${ }^{62,63}$ we applied the optical SHG technique to study the electrochemical oxidation of formaldehyde on a Pt electrode at $532 \mathrm{~nm}$ excitation and found that the SHG response was sensitive to the existence of $\mathrm{CO}(\mathrm{a})$. We proposed that the electron transition resonance was important in the SHG of this particular case, although it was not clear whether the fundamental photon or the SH photon was energetically coupled with the electronic transition and what states were associated with the resonance.

In this paper, we carried out wavelength dependent SHG experiments of $\mathrm{CO}(\mathrm{a})$ on a Pt electrode at several excitation wavelengths and examined the resonance scheme making reference to the UHV studies in order to clarify the mechanisms for the SHG enhancement. SFG measurements were also carried out to extend the wavelength region to be investigated.

\section{Experimental Section}

Nonlinear Optical Measurement. Two different light sources were used to create a nonlinear optical (NLO) signal on the electrode surface. One was a $10 \mathrm{~Hz}$ Q-switched $\mathrm{Nd}$ : YAG laser (LEXEL, HY-200S), and the other was an optical parametric oscillator (OPO: Lambda Physik, Scan Mate OPPO) pumped by third-harmonic light $(354.8 \mathrm{~nm})$ from a dipolepumped Nd:YAG laser (Coherent, Infinity 40-100). The NLO spectrum over the broad photon energy range was obtained by combining two different interfacial nonlinear optical methods. SHG measurement with the signal light $(520 \mathrm{~nm}<\omega<680$ $\mathrm{nm}$ ) from the OPO provided information within the energy range $260 \mathrm{~nm}<2 \omega<340 \mathrm{~nm}$, and SFG measurement with the signal light $\left(520 \mathrm{~nm}<\omega_{1}<640 \mathrm{~nm}\right)$ from the OPO and fundamental light $\left(\omega_{2}=1064 \mathrm{~nm}\right)$ from the YAG laser provided information in the energy range $350 \mathrm{~nm}<\omega_{1}+\omega_{2}<400 \mathrm{~nm}$. SFG measurement covered the wavelength region where the intensity of visible light from the OPO was too low to create the detectable SHG signal. SHG measurement with the fundamental light $(1064 \mathrm{~nm})$ from the YAG laser was also carried out to measure a near-infrared excited SHG response.

Figure 1a shows the experimental arrangement for the SHG measurement for 1064 and $532 \mathrm{~nm}$ excitation. ${ }^{62-64}$ The excitation beam was slightly focused onto the surface of a platinum electrode in a spectroelectrochemical cell with angle of incidence at $60^{\circ}$. The fluencies of the excitation were $<2$ $\mathrm{mJ} / \mathrm{pulse}$ for the visible excitation and $<10 \mathrm{~mJ} / \mathrm{pulse}$ for the near-infrared excitation to obtain good signal/noise ratios. The energy density of excitation pulses was much smaller than the damage threshold of platinum surfaces. The $\mathrm{SH}$ light was separated from the excitation laser beam with proper shortwavepass glass filters and a spectrometer (Ritu, MC-10N) and detected by a photomultiplier tube (Hamamatsu, R636). The intensity of SH light pulses was averaged with a boxcar averager (Stanford Research, SR250). The intensity and temporal profile of the excitation beam were monitored by employing a separated reference channel. The reference channel consisted of a BBO crystal (Cleveland Crystals), filters, and a fast photodiode. The partially $(<10 \%)$ reflected excitation beam was doubled in the BBO crystal and the intensity of generated SH light was averaged with another boxcar averager. The SHG signal was normalized to the excitation light intensity. The polarization of the excitation beam was set with a combination of a polarizer and a double Fresnel rhomb, and the polarization of the $\mathrm{SH}$ light was selected by another polarizer. All the nonlinear optical data reported in this paper were obtained with the $p$-in/p-out polarization condition.

Experimental arrangements for the SHG and SFG measurements using the OPO/YAG light source were essentially the same as that described above with minor differences. In these cases, the square of the intensity of the partially reflected fundamental light was used as a reference signal. A combination of a double Fresnel rhomb and a Glan-laser polarizer was used not only to set the input polarization but also to control the input laser power at various wavelengths from the OPO to be constant. The fluencies of the excitation were $<2 \mathrm{~mJ} /$ pulse for the visible SHG measurement. In the case of the SFG measurement, the near-infrared beam from the YAG laser was set on the same beam path of the visible beam from OPO by means of the proper harmonic separation mirrors, and sum fluencies of the excitation beams were $<3 \mathrm{~mJ} /$ pulse.

Electrochemistry. A $10 \mathrm{~mm}$ diameter platinum disk held in a KeI-F holder (the apparent electrode diameter: $8 \mathrm{~mm}$ ) was used for the electrochemical NLO measurement. This electrode was polished with alumina powder, which was exchanged successively to smaller grades down to $0.05 \mu \mathrm{m}$, to be optically flat and was sonicated in deionized water before it was introduced in a spectroelectrochemical cell.

Water purified by the Milli-Q system and as-received perchloric acid (reagent grade, Wako Pure Chemicals) were used to prepare the electrolyte solution. Deaeration of the solution was carried out by bubbling the high-purity nitrogen $(>99.99 \%)$ through the electrolyte solution. CO-saturated solution was prepared by passing $\mathrm{CO}$ gas through the electrolyte solution in the spectroelectrochemical cell. The electrode potential was controlled with an electric polarization unit (Toho Tech. Res., PS-07). The electrode potential, the current, and the normalized SHG signal were converted to digital values via an a/d converter and stored on a personal computer (NEC, PC-9801RS).

A standard three-electrode configuration was constructed with an $\mathrm{Ag} / \mathrm{AgCl}$ (saturated $\mathrm{NaCl}$ ) reference electrode and a $\mathrm{Pt}$ counter electrode. After the introduction of the working electrode to the spectroelectrochemical cell, the electrochemical cleaning cycles, which consisted of continuous potential cycling between -0.2 and $+1.2 \mathrm{~V}$ in $0.1 \mathrm{M} \mathrm{HClO}_{4}$ solution, were performed before each electrochemical SHG experiment until the cyclic voltammogram (CV) became that of a clean platinum electrode.

The experimental sequence for the electrochemical NLO experiments was as follows and is described in Figure 1b. In the course of the potential sweep in the $0.1 \mathrm{M} \mathrm{HClO}_{4}$ solution, the potential scan was stopped at $E_{\text {ads }}$, and then, $\mathrm{CO}$ gas was introduced to the spectroelectrochemical cell through a Teflon tube. After $5 \mathrm{~min}, \mathrm{CO}$ remaining in the solution was removed by bubbling nitrogen gas through the electrolyte solution for $15 \mathrm{~min}$, and then, the potential was swept in the positive direction. Consequently, $\mathrm{CO}$ should exist only on the platinum surface at the initial stage of the experiments and the influence on the NLO response by the adsorption of excess CO was avoided.

\section{Results}

Figure 2 shows typical results of the cyclic voltammogram (a) and the potential dependent profiles of the SHG signal excited at $532 \mathrm{~nm}$ (b) and $1064 \mathrm{~nm}$ (c) at a bare Pt electrode in 
(a)

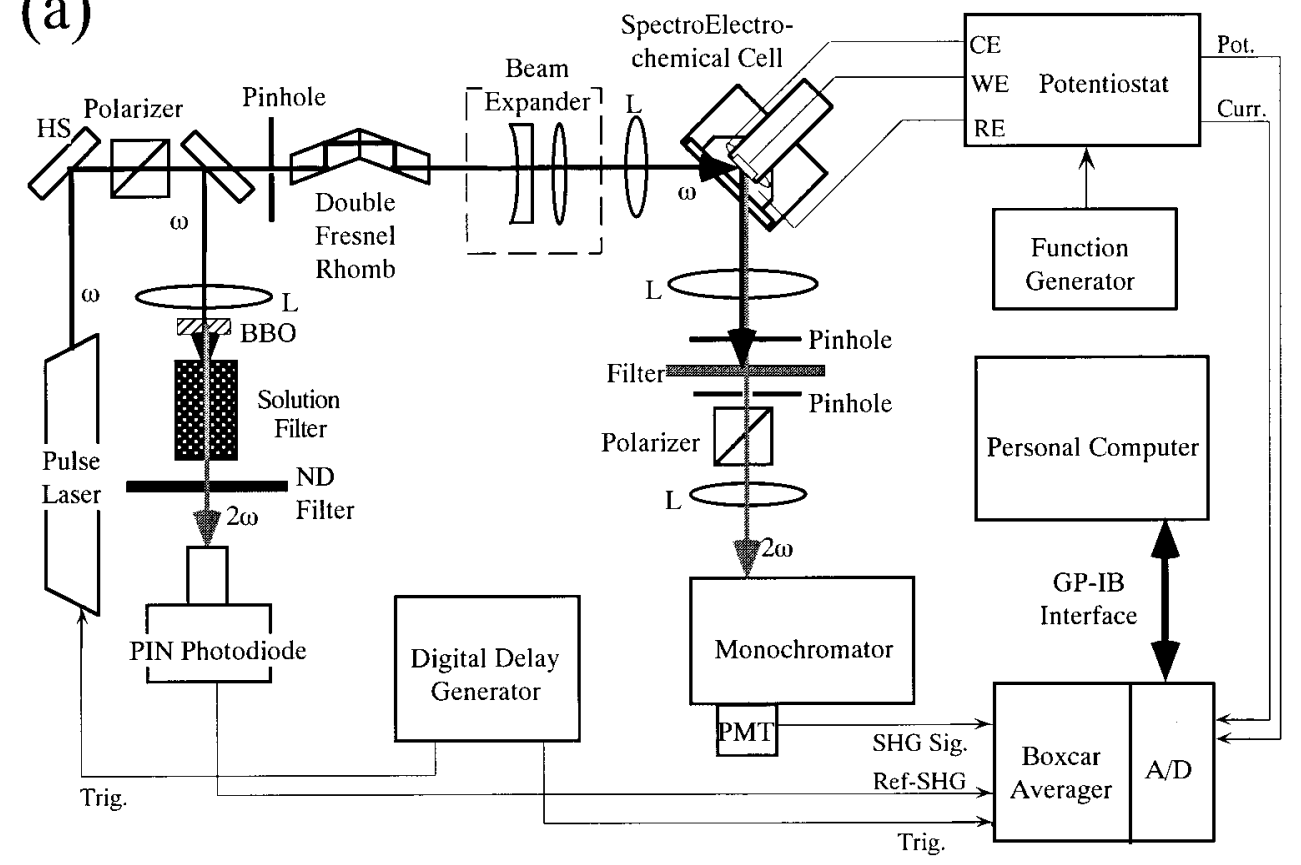

(b)

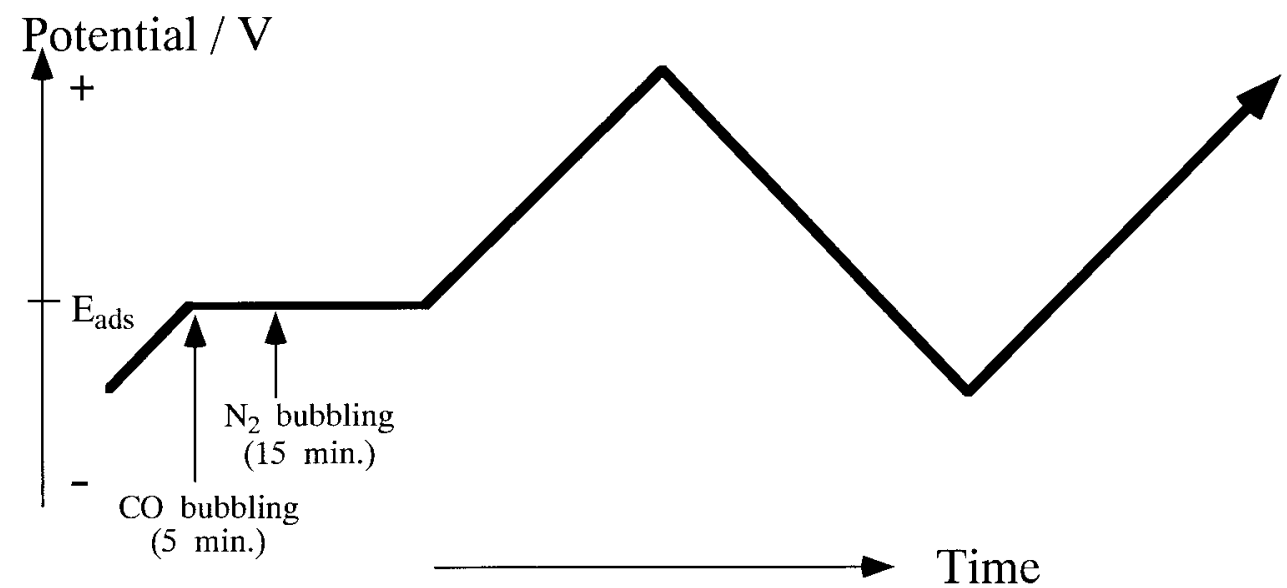

Figure 1. (a) Experimental apparatus for optical SHG measurement. L:lens. (b) The potential scheme of the electrochemical SHG measurement for the Pt electrode with preadsorbed CO.

$0.1 \mathrm{M} \mathrm{HClO}_{4}$ solution. At both excitation wavelengths, the enhancement of SHG with the formation of underpotentially deposited hydrogen (upd-H) in the negative potential region was observed. These potential dependences are similar to that obtained at Pt electrodes with $604 \mathrm{~nm}$ excitation by Corn and his co-workers. ${ }^{65,66}$ They introduced the resonant mechanism idea to explain the SHG enhancement with the adsorption of hydrogen on Pt. Although a similar potential dependent profile of SHG was obtained for $1064 \mathrm{~nm}$ excitation (Figure 2d), the input light power should be at least 3 times larger than that for the $532 \mathrm{~nm}$ excitation measurement to obtain a reasonable signal/noise ratio. In addition, the minor difference between the SHG behaviors for each excitation wavelength was observed in the platinum oxide formation region.

Figure 3 shows (a) the cyclic voltammogram and (b) the potential dependence of the $1064 \mathrm{~nm}$-excited SHG signal (532 nm) with $E_{\text {ads }}=-0.2 \mathrm{~V}$. In the first anodic scan (solid line), the current corresponding to the formation of upd-H was not found and two distinct $\mathrm{CO}$ oxidation peaks were observed at +0.2 and $+0.38 \mathrm{~V}$. The small peak around $+0.2 \mathrm{~V}$ and the peak around $+0.38 \mathrm{~V}$ are referred to as a prepeak and a main peak, respectively. ${ }^{5}$ It is well-known that the peak potential and the shapes of these peaks depend on $\mathrm{pH}$, anion species, sweep rate, and $E_{\text {ads. }}{ }^{5,8,19,67,68}$ The shape of the $\mathrm{CV}$ after the main peak is very similar to that from the bare Pt electrode (Figure 1a). The voltammogram for the second potential scan, represented by a dotted line, showed the hydrogen waves and no trace of the $\mathrm{CO}$ oxidation peaks. In the first anodic sweep, the SHG signal from the $\mathrm{CO}(\mathrm{a}) / \mathrm{Pt}$ surface (solid line) decreased monotonically as the potential was scanned from $-0.2 \mathrm{~V}$ to more positive potentials and became almost the same value as that of the bare Pt electrode before the main oxidation peak of $\mathrm{CO}(\mathrm{a})$. The SHG response in the second scan was similar to that observed at the bare Pt electrode, represented by a broken line in Figure 2c. The SHG signal from the CO/Pt surface in the potential region more negative than $+0.35 \mathrm{~V}$ was larger than that from the bare $\mathrm{Pt}$ surface in the double-layer region $(+0.05 \mathrm{~V}$ to $+0.4 \mathrm{~V})$. The enhancement of the SHG signal from the $\mathrm{CO}$-covered $\mathrm{Pt}$ electrode should not be due to upd-H, as no hydrogen waves were found in the voltammogram of the first anodic scan, apparently due to the existence of the $\mathrm{CO}(\mathrm{a})$ monolayer. 


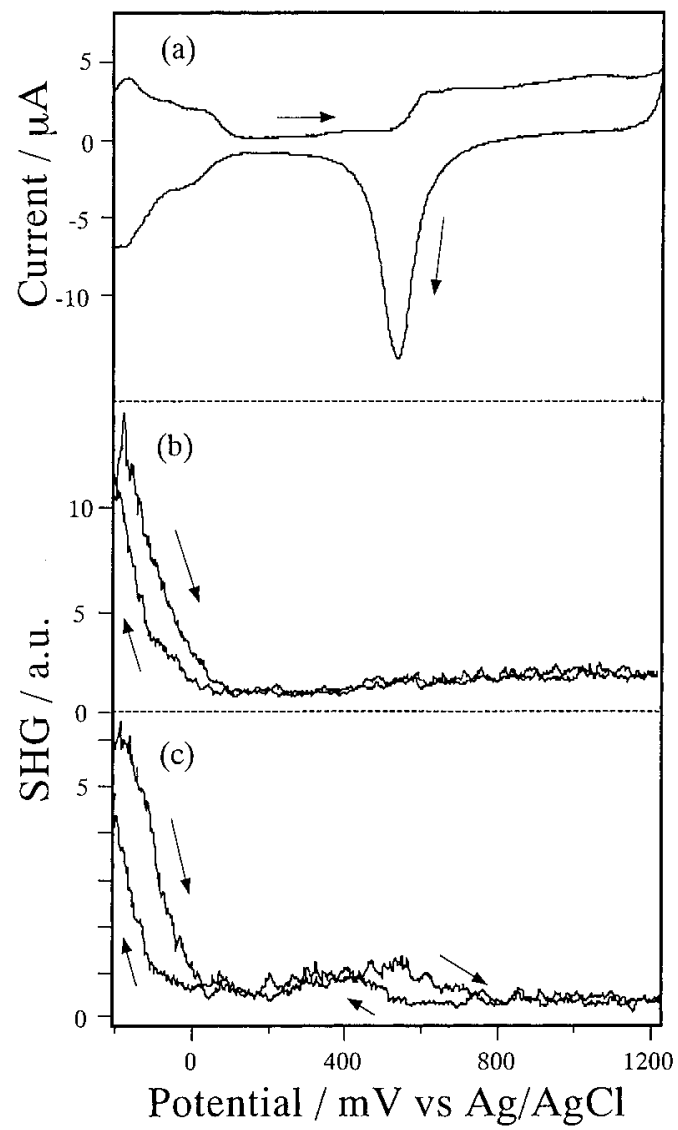

Figure 2. Cyclic voltammograms of a Pt electrode in $0.1 \mathrm{M} \mathrm{HClO}_{4}$ (a) and simultaneously recorded potential dependences of $\mathrm{p}$-in/p-out SHG signal $(266 \mathrm{~nm})$ at $532 \mathrm{~nm}$ excitation (b) and that $(532 \mathrm{~nm})$ at $1064 \mathrm{~nm}$ excitation (c). Sweep rate: $5 \mathrm{mV} \mathrm{s}^{-1}$.

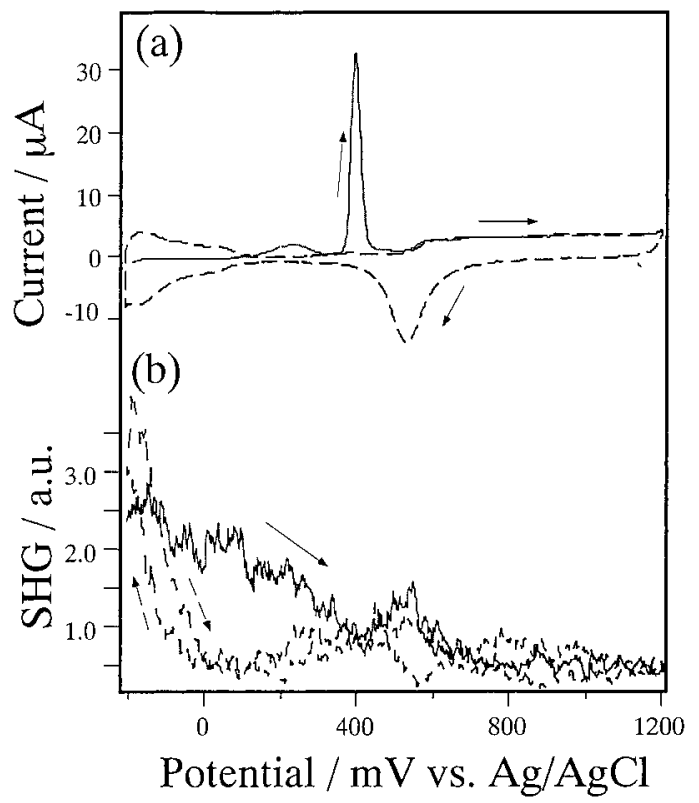

Figure 3. Potential dependence of (a) current and (b) p-in/p-out SHG signal $(532 \mathrm{~nm})$ at a Pt electrode with preadsorbed $\mathrm{CO}$ during the first two cycles of potential scan. Solid and broken lines represent the first and second scan, respectively. Excitation wavelength: $1064 \mathrm{~nm}$. Sweep rate: $5 \mathrm{mV} \mathrm{s}^{-1}$.

Figure 4 shows typical results of (a) the cyclic voltammogram and (b) the potential dependent profile of the $532 \mathrm{~nm}$-excited SHG signal $(266 \mathrm{~nm})$ for the first two potential cycles after the preadsorption of $\mathrm{CO}$ for $E_{\mathrm{ads}}=-0.2 \mathrm{~V}$. While the voltammetric response was the same as that of Figure $3 \mathrm{a}$ as expected, the

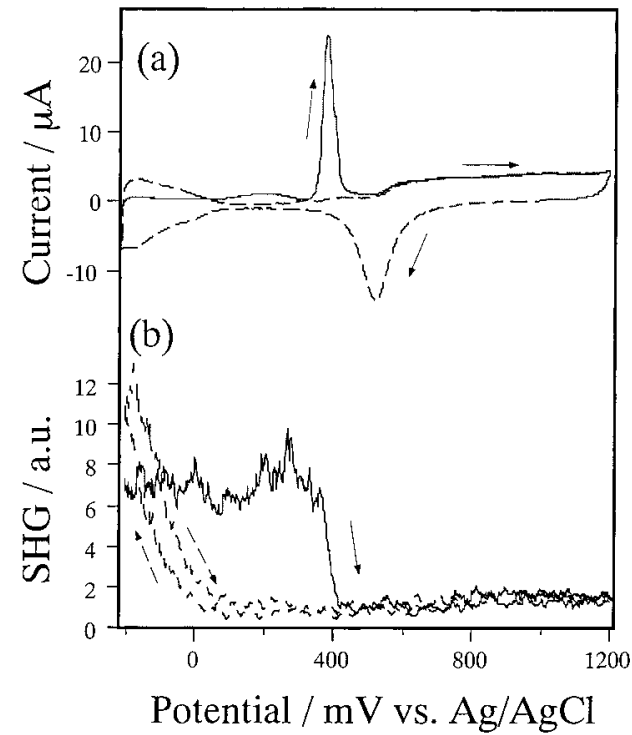

Figure 4. Potential dependence of (a) current and (b) p-in/p-out SHG signal $(266 \mathrm{~nm})$ at a Pt electrode with preadsorbed CO during the first two cycles of potential scan. Solid and broken lines represent the first and second scan, respectively. Excitation wavelength: $532 \mathrm{~nm}$. Sweep rate: $5 \mathrm{mV} \mathrm{s}^{-1}$.

potential dependence of the SHG signal was different from that of the near-infrared excitation (Figure 3b). The SHG behavior seemed to correspond well to the features of the cyclic voltammogram in contrast to the results in Figure 3. The SHG signal was much higher than that of the bare Pt electrode in the double-layer region and was fairly constant in the potential region more negative than the main oxidation peak of $\mathrm{CO}(\mathrm{a})$. The SHG signal did not change with the prepeak. It decreased irreversibly with the main oxidation peak of $\mathrm{CO}(\mathrm{a})$ in the first scan and became the same as that of the bare Pt electrode. Only the enhancement of the SHG with the formation of upd-H in the negative potential region was reversively observed, and the potential dependence of the SHG signal after the second scan was almost identical to that in Figure 2. Essentially the same SHG behavior was observed by visible excitation in the wavelength range between 520 and $680 \mathrm{~nm}$.

To confirm that the enhancement of the SHG signal with visible excitation is due to $\mathrm{CO}(\mathrm{a})$, the effect of $E_{\text {ads }}$ on the potential dependence of the SHG signal was investigated. In general, the potential where the oxidation of $\mathrm{CO}(\mathrm{a})$ occurs shifts positive with $E_{\text {ads }}$ being more positive. Figure 5 shows the potential dependence of (a) the current and (b) the $650 \mathrm{~nm}$ excited SHG signal $(325 \mathrm{~nm})$ with $E_{\text {ads }}=-0.15 \mathrm{~V}$ (solid line) and $E_{\text {ads }}=+0.15 \mathrm{~V}$ (broken line). The essential features of these results were similar. The SHG signal was higher than that at the bare Pt electrode and was fairly constant in the potential region where the $\mathrm{Pt}$ surface was covered with $\mathrm{CO}$. The SHG signal decreased as soon as $\mathrm{CO}$ (a) oxidation current flowed. The position of the main oxidation peak of the latter $(+0.46 \mathrm{~V})$ was a little positive of the former $(+0.41 \mathrm{~V})$, as expected, and correspondingly, the SHG signal started to decrease at the more negative potential in the former. Thus, it was concluded that the SHG signal at $650 \mathrm{~nm}$ excitation was enhanced by the existence of $\mathrm{CO}(\mathrm{a})$ that was oxidized at the main oxidation peak.

Figure 6 shows (a) the cyclic voltammogram and (b) the potential dependence of the $1064 \mathrm{~nm}$ - and $580 \mathrm{~nm}$-excited SFG signals $(375 \mathrm{~nm})$ with $E_{\text {ads }}=-0.15 \mathrm{~V}$. The SFG signal was fairly constant in the potential region more negative than the main oxidation peak of $\mathrm{CO}(\mathrm{a})$ in the first cycle. A large enhancement of the SFG signal by upd-H was apparent in the 


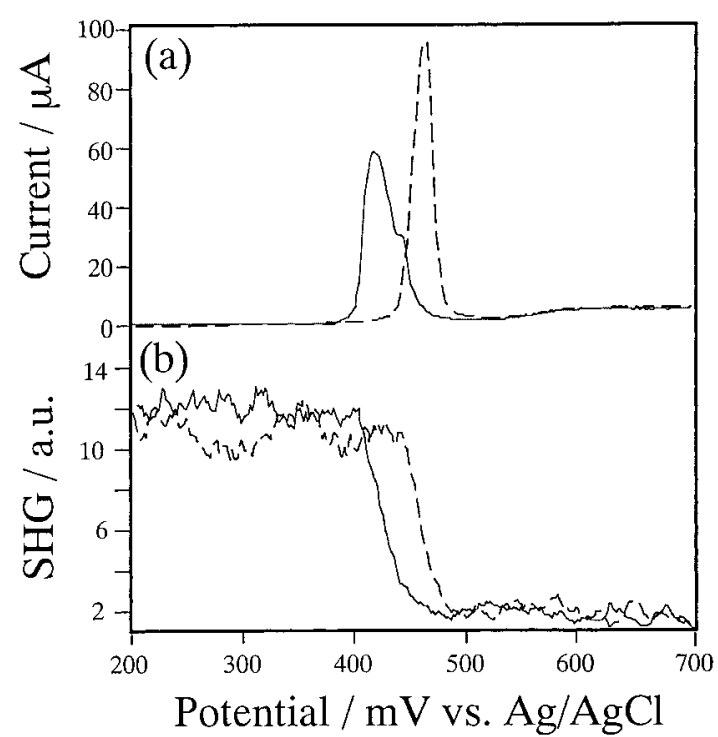

Figure 5. Potential dependence of (a) current and (b) p-in/p-out SHG signal $(325 \mathrm{~nm})$ at a Pt electrode and preadsorbed $\mathrm{CO}$ during the fist anodic potential scan with $E_{\text {ads }}=-0.15 \mathrm{~V}$ (solid line) and $E_{\text {ads }}=+0.15$ $\mathrm{V}$ (broken line). Excitation wavelength: $650 \mathrm{~nm}$. Sweep rate: $5 \mathrm{mV}$ $\mathrm{s}^{-1}$.

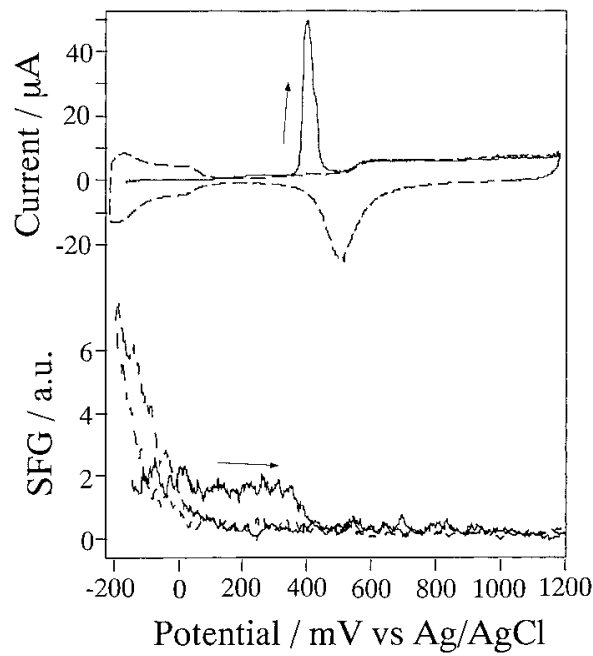

Figure 6. Potential dependence of (a) current and (b) p-in/p-out SFG signal $(375 \mathrm{~nm})$ at a Pt electrode and preadsorbed CO during the fist two cycles of potential scan. Solid and broken lines represent the first and second scan, respectively. Excitation wavelength: 1064 and 580 $\mathrm{nm}$. Sweep rate: $10 \mathrm{mV} \mathrm{s}^{-1}$.

negative potential region of the second potential scan. Although this behavior was very similar to that of the $532 \mathrm{~nm}$-excited SHG shown in Figure 4, the enhancement of the SFG signal by $\mathrm{CO}$ (a) was much smaller than the SHG enhancement in Figure 4. Furthermore, the enhancement of $290 \mathrm{~nm}$ light, i.e., SHG at $580 \mathrm{~nm}$ excitation, by $\mathrm{CO}(\mathrm{a})$ on a Pt surface was much larger, although the potential dependent NLO behavior was the same as that of the $375 \mathrm{~nm}$ SFG signal. Nevertheless, the SFG behavior in Figure $6 \mathrm{~b}$ was totally different from the SHG behavior $(532 \mathrm{~nm})$ for $1064 \mathrm{~nm}$ excitation shown in Figure 3b, where the SHG signal decreased monotonically as potential became more positive. These results suggest that these differences in the enhancement were caused by the difference in the NLO wavelengths of generated photons. Consequently, the energy range where the enhancement of nonlinear optical signal by the existence of $\mathrm{CO}$ (a) occurs was more localized, perhaps in the UV region, than that by the existence of upd-H.

The NLO signal was apparently enhanced by the adsorption of $\mathrm{CO}(\mathrm{a})$ on the Pt surface for all the excitation wavelengths

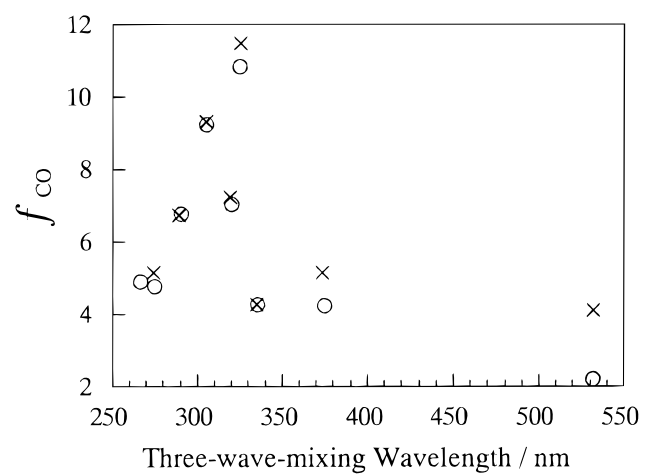

Figure 7. NLO enhancement factor, $f_{\mathrm{CO}}$, as a function of the threewave-mixing wavelength for a Pt surface with preadsorbed $\mathrm{CO}$ at +0.15 $\mathrm{V}(\mathrm{O})$ and at $-0.15 \mathrm{~V}(\times)$. Details are described in the text.

employed here. The electrochemical NLO behaviors were, however, different for visible excitation and for near-infrared excitation. To quantify the enhancement characteristics, we define the enhancement factor, $f_{\mathrm{CO}}$, as the ratio

$$
f_{\mathrm{CO}}=\frac{I\left(\omega_{1}+\omega_{2}\right)}{I\left(\omega_{1}+\omega_{2}\right)_{0}}
$$

where the $I\left(\omega_{1}+\omega_{2}\right)$ is the NLO signal from the CO-covered platinum surface at a given potential and $I\left(\omega_{1}+\omega_{2}\right)_{0}$ is the NLO signal from the bare platinum surface at $+0.05 \mathrm{~V}$. In the case of SHG, $\omega_{1}=\omega_{2}=\omega$, i.e., $\omega_{1}+\omega_{2}=2 \omega$. Figure 7 shows the wavelength dependence of $f_{\mathrm{CO}}$ at -0.15 and $+0.15 \mathrm{~V}$. Although $f_{\mathrm{CO}}$ is larger than unity in all the cases, the enhancement is more significant when visible light was employed for excitation. The most significant difference is, however, while $f_{\mathrm{CO}}$ was independent of the potential for visible excitation, $f_{\mathrm{CO}}$ was higher at negative potential for near-infrared excitation, as already mentioned. Thus, the mechanism for the enhancement of the NLO signal should be different for each excitation wavelength.

\section{Discussion}

The SHG characteristics of $\mathrm{CO}(\mathrm{a})$ on Pt have been investigated in UHV. ${ }^{69}$ The exposure to $\mathrm{CO}$ induced a rather complex change of SHG signal at the Pt surface depending on the CO pressure in UHV. The $532 \mathrm{~nm}$-excited $\mathrm{SHG}$ signal from a $\mathrm{Pt}$ surface first increased by the exposure of CO. After it reached the maximum, which is only ca. $120 \%$ of the SHG signal from the bare platinum surface, it started to decrease with the increase of the $\mathrm{CO}$ pressure. It increased again with further increase of the $\mathrm{CO}$ pressure. In contrast, the $532 \mathrm{~nm}$-excited SHG signal from the $\mathrm{CO}$-covered $\mathrm{Pt}$ surface was 4 times larger than that from the bare Pt surface in the electrochemical environment, and the coverage dependence of the SHG signal was rather simple; that is, the SHG signal monotonically decreased as CO(a) was desorbed. Bae obtained similar results in an electrochemical environment and discussed the possible cause of the differences of SHG response between the two environments. ${ }^{70}$ The coverage dependence of the NLO signal is discussed subsequently. While the SHG signal by near-infrared excitation decreased by exposure to $\mathrm{CO}$ in the UHV, it also increased by adsorption of $\mathrm{CO}$ in the electrochemical environment. Thus, the NLO characteristics of $\mathrm{CO}(\mathrm{a})$ on $\mathrm{Pt}$ in electrolyte solutions are different from those in UHV conditions.

The General Treatment of the NLO Signal. The NLO light intensity arising from a metal surface has been treated in detail by several authors ${ }^{32,71-74}$ and is briefly described here. At a metal-solution interface, the induced surface nonlinear polar- 
ization, $P\left(\omega_{1}+\omega_{2}\right)$, is given by eq 2 in the electric dipole approximation. $^{32}$

$$
P\left(\omega_{1}+\omega_{2}\right)=\chi_{\mathrm{S}}^{(2)}: E\left(\omega_{1}\right) E\left(\omega_{2}\right)
$$

where $\chi_{\mathrm{s}}{ }^{(2)}$ is the surface nonlinear susceptibility and $E\left(\omega_{1}\right)$ and $E\left(\omega_{2}\right)$ are the incident electric fields due to the light with a frequency of $\omega_{1}$ and $\omega_{2}$, respectively. The surface nonlinear susceptibility is changed by the presence of an adsorbate and becomes a new quantity, $\chi^{\prime} s^{(2)}$ :

$$
\chi_{\mathrm{s}}^{(2)}=\chi_{\mathrm{S}}^{(2)}+\chi_{\mathrm{A}}^{(2)}+\Delta \chi_{\mathrm{I}}^{(2)}
$$

where $\chi_{\mathrm{A}}^{(2)}$ is the inherent nonlinear susceptibility of the adsorbate itself which is independent of the surface and $\Delta \chi_{\mathrm{I}^{(2)}}^{(2)}$ is the perturbation in nonlinear susceptibility due to the interactions between the surface and the adsorbate. While $\chi_{\mathrm{A}}^{(2)}$ is usually very small compared to $\chi_{\mathrm{S}}{ }^{(2)}$ for metal surfaces, $\Delta \chi_{\mathrm{I}}{ }^{(2)}$ may be large to affect $\chi^{\prime} s^{(2)}$ by adsorption.

The additional contribution to the NLO signal from an electrode surface that must be considered is a nonlinear susceptibility term induced by the dc electric field..$^{33}$ By taking this term into account, the surface nonlinear susceptibility is given by

$$
\chi_{\mathrm{S}}^{\prime \prime(2)}=\chi_{\mathrm{s}}^{(2)}+\chi_{\mathrm{DC}}{ }^{(3)} E_{\mathrm{DC}}
$$

where $\chi_{\mathrm{DC}}^{(3)}$ is the third-order nonlinear hyperpolarizability tensor for the metal surface and $E_{\mathrm{DC}}$ is the static electric field at the metal/electrolyte interface. This dc effect, however, is difficult to distinguish from the electronic perturbation effect, i.e., $\Delta \chi_{\mathrm{I}}^{(2)}$, which is caused by the reorganization of the electronic structure of the bonding between the surface and the adsorbate when strongly adsorbed anions exist. Therefore, any changes in the NLO response as a function of electrode potential must be considered to be due to the combination of both effects.

SHG Behaviors with Near-IR Excitation: dc Electric Field Induced SHG. The SHG signal from the $\mathrm{CO}(\mathrm{a}) / \mathrm{Pt}$ surface for near-infrared excitation decreased monotonically as the electrode potential became more positive, although the coverage of CO(a) was constant in this potential region $(-0.2$ to $+0.38 \mathrm{~V}$, Figure 3). This result suggests that the enhancement is not due to resonance with $\mathrm{CO}$ (a) related states. However, since such a potential dependent SHG behavior of a Pt electrode was not observed in this potential region, unless the surface was covered by $\mathrm{CO}$ (a) (Figure 1), $\mathrm{CO}$ (a) certainly plays a crucial role in SHG. The monotonic change of the SHG signal with potential suggests that this is dc electric field induced SHG (EISHG) at the electrochemical interfaces. ${ }^{49-52,74}$ This effect is expressed by eq 4 , and the SHG signal is expected to increase with increase of the surface charge. The potential dependence of EISHG was well studied at roughened $\mathrm{Ag}$ electrode/electrolyte interfaces ${ }^{49-52}$ and was nearly parabolic, i.e., $I_{2 \omega}(\phi) \propto\left(\phi-\phi_{0}\right)^{2} \approx E_{\mathrm{DC}^{2}}$, where $\phi$ and $\phi_{0}$ are the electrode potential and the potential of zero charge (pzc). At the CO/Pt surface, it was expected that negative surface charge decreases with a positive-going potential scan in the potential region concerned because the pzc's at lowindex Pt surfaces with saturated $\mathrm{CO}$ adlayers were determined to be around $0.8-1.0 \mathrm{~V}$ vs SCE. ${ }^{25-27}$ These values are much more positive than the potential region where SHG enhancement was observed (Figure 3). EISHG was not observed in the double-layer region at the bare Pt electrode, as shown by the broken line of Figure $2 b$. This is due to the fact that the pzc of the bare Pt electrode is $+0.3 \mathrm{~V}$ vs SHE ${ }^{75}$ which is within the double-layer region, and therefore, the charge increase should be rather small within the potential region concerned. Further- more, the EISHG effect is hidden in the negative potential region due to the resonant enhancement by hydrogen absorption and is disturbed by oxide formation in the positive potential region. Adsorption of $\mathrm{CO}$ causes expansion of the potential windown, providing favorable circumstances to observe the EISHG effect.

NLO Behaviors with Visible Excitation: Resonant Enhancement. NLO enhancement with visible excitation was much higher than that with near-infrared excitation and depended on the amount of $\mathrm{CO}(\mathrm{a})$. If the nonresonant contribution is dominant for all the cases examined here, the wavelength dependence of the SHG must be small. The rather large wavelength dependence of the NLO response shown in Figure 7 suggests a relatively large contribution of the resonant NLO effect for the enhancement. The NLO enhancement in the resonance regime appears as a result of an energetic coupling between the photon associated with the NLO process and the electron transition. ${ }^{76}$ Since the resonant NLO enhancement can be observed when either the fundamental photon or the NLO photon is coupled with the electron transition, the determination of the resonant photon energy is difficult using SHG measurements with a single excitation wavelength.

Since the SHG enhancement at the $1064 \mathrm{~nm}$-input/532 nmoutput condition was nonresonant but the $532 \mathrm{~nm}$-input $/ 266 \mathrm{~nm}$ output condition was resonant as described above, not visible photons but ultraviolet photons above $3.5 \mathrm{eV}$ should be coupled with a transition. If visible photons are coupled with the transition, the SHG response at the $1064 \mathrm{~nm}$-input/532 nmoutput condition should be similar to that at the $532 \mathrm{~nm}$-input/ $266 \mathrm{~nm}$-output condition. The results of SFG presented in Figure 6 also support the resonance of the ultraviolet photon. Under 1064 and $580 \mathrm{~nm}$ excitations, the SFG signal $(375 \mathrm{~nm})$ was not enhanced by $\mathrm{CO}$ (a) as much as the SHG signal (290 $\mathrm{nm}$ ). If the visible photon contributed to the resonance, the enhancement of $1064 \mathrm{~nm}$ - and $580 \mathrm{~nm}$-excited SFG signals by $\mathrm{CO}$ (a) should be comparable to that of the SHG signal in Figure 4 and Figure 5.

Additional confirmation of the coupling in the UV region was given by studies with linear optics. ${ }^{77-81}$ An absorption peak around $270 \mathrm{~nm}(4.59 \mathrm{eV})$ was observed for the CO/Pt surface by potential-modulated reflectance spectroscopy (PMRS) and differential reflectance spectroscopy. This absorption was attributed to charge transfer transition of the $\mathrm{CO}-\mathrm{Pt}$ surface complex, although a detailed description was not provided. The electronic structure of the $\mathrm{CO} / \mathrm{Pt}$ surface has been well investigated with electron spectroscopic techniques in UHV environment. According to ultraviolet photoemission spectroscopy (UPS) studies, ${ }^{14,15}$ occupied states originating from $4 \sigma$ and $5 \sigma_{\mathrm{b}}+1 \pi$ molecular states of $\mathrm{CO}$ are found to be positioned around 11.8 and $9.2 \mathrm{eV}$, respectively, below the Fermi energy $\left(E_{\mathrm{f}}\right)$. Inverse photoemission spectroscopy (IPS) studies ${ }^{16-18}$ showed that two unoccupied states are located at 1.5 and 4.5 $\mathrm{eV}$ above $E_{\mathrm{f}}$, which are assigned to the antibonding $5 \sigma_{\mathrm{a}}$ and $2 \pi_{\mathrm{a}}{ }^{*}$, respectively. Fukutani et al. ${ }^{82,83}$ summarized these results and showed the energies of the CO/Pt system, but they did not mention any states related to $\mathrm{CO}$ (a) near the Fermi level. Schmidt et al ${ }^{84}$ examined the electronic states near the Fermi level by measuring the potential dependence of the $\mathrm{CO}$ stretching vibration lifetime with time-resolved IR-visible sum frequency generation technique and showed a rough sketch of the density of $2 \pi^{*}$-derived states. The energy diagram for the $\mathrm{CO} / \mathrm{Pt}$ system is schematically shown in Figure 8, combining the results of refs $82-84$.

As for the SHG enhancement, the lower transition threshold may be around $3.5 \mathrm{eV}$ (see Figure 7). Most of the electronic transitions considered for the CO/Pt (111) surface, ${ }^{85}$ e.g., $5 \sigma_{\mathrm{b}}$ 


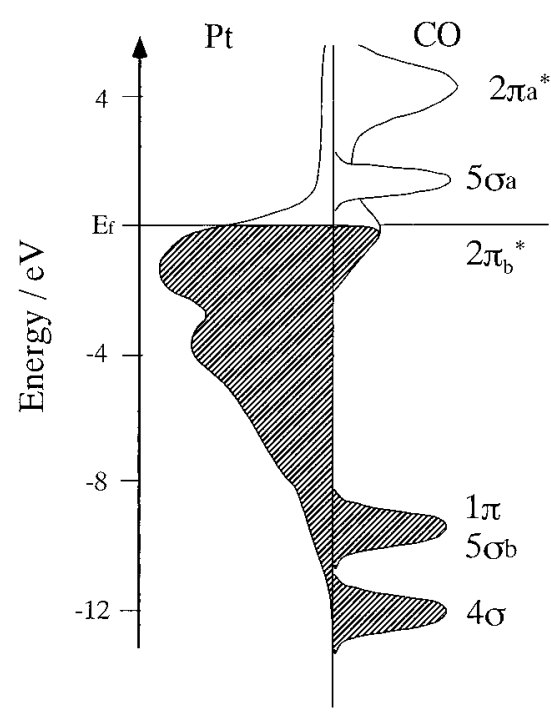

Figure 8. Schematic energy diagram and assignments of occupied and unoccupied states of $\mathrm{Pt}$ with adsorbed $\mathrm{CO}$.

$\rightarrow 2 \pi_{\mathrm{a}}{ }^{*}, 1 \pi \rightarrow 5 \sigma_{\mathrm{a}}$, and from $1 \pi$ to the surface states around $E_{\mathrm{f}}$, are ruled out as responsible for the resonance because photon energies required for these transitions are too high, even if the final state effects are taken into account for the experimentally determined binding energy. The transition of a Pt electron around $E_{\mathrm{f}}$ to the $5 \sigma_{\mathrm{a}}$ state mentioned in ref 70 can also be ruled out because the photon energy for this transition is too small. The only possible transition responsible for the SHG resonance is from Pt electronic states around $E_{\mathrm{f}}$ to the $2 \pi_{\mathrm{a}}{ }^{*}$ state directly or indirectly via the Pt s-band, whose energy is ca. $4.5 \mathrm{eV}$. Thus, we can conclude that the SHG enhancement at visible excitation is due to the coupling of ultraviolet SH photons with the metalligand charge transfer.

The coverage dependence of the NLO signal should provide information on the nature of the adsorbate. As shown in eq 3, the effect of $\mathrm{CO}$ absorption on NLO response should be due to $\chi_{\mathrm{A}}^{(2)}$ or/and $\Delta \chi_{\mathrm{I}}{ }^{(2)}$. By using the molecular nonlinear susceptibility of $\mathrm{CO}, \beta=129 \times 10^{-33} \mathrm{esu},{ }^{86,87}$ the inherent nonlinear susceptibility for the close-packed monolayer of $\mathrm{CO}$ can be estimated as $\chi_{\mathrm{A}}^{(2)} \approx 10^{-16}-10^{-17}$ esu. This is negligible compared with the nonlinear susceptibilities of metal surfaces, $\chi_{\mathrm{S}}{ }^{(2)}$, which are on the order of $10^{-13}$ esu. ${ }^{88}$ Thus, the $\Delta \chi_{\mathrm{I}}{ }^{(2)}$ term must be dominant in the NLO response induced by $\mathrm{CO}$ adsorption. Since the nonlinear susceptibility of the surface changes with the surface coverage of $\mathrm{CO}(\mathrm{a})$, the NLO signal should be represented by a linear function of the surface concentration of $\mathrm{CO}(\mathrm{a}), \Gamma_{\mathrm{CO}}$, only when $\Delta \chi_{\mathrm{I}}^{(2)}$ is proportional to $\Gamma_{\mathrm{CO}}$.

$$
\chi_{\mathrm{s}}^{(2)}=\chi_{\mathrm{s}}^{(2)}\left(1+c \gamma_{\mathrm{CO}}\right)
$$

where $c$ is a proportionally constant, which is related to $\Delta \chi_{\mathrm{I}}^{(2)}$. In this case, the following correlation is derived, since the NLO signal is proportional to the square of $\chi^{\prime} \mathrm{s}^{(2)}$ and $\Gamma_{\mathrm{CO}}$ is derived from the Faradaic charge density $Q_{\mathrm{CO}}$ is associated with the oxidation of $\mathrm{CO}(\mathrm{a})$ :

$$
\frac{\chi_{\mathrm{S}}^{(2)}}{\chi_{\mathrm{S}}^{(2)}} \propto\left(\frac{I\left(\omega_{1}+\omega_{2}\right)}{I\left(\omega_{1}+\omega_{2}\right)_{0}}\right)^{1 / 2}=A+c^{\prime} Q_{\mathrm{CO}}
$$

where $I\left(\omega_{1}+\omega_{2}\right)_{0}$ is the NLO signal from the bare platinum surface and $A$ and $c^{\prime}$ are the proportionality constants that are introduced by the modification of eq 5 into eq 6 . Figure 9 shows the $\left(I(2 \omega) / I(2 \omega)_{0}\right)^{1 / 2}$ - charge density $\left(Q_{\mathrm{CO}}\right)$ plot for the CO

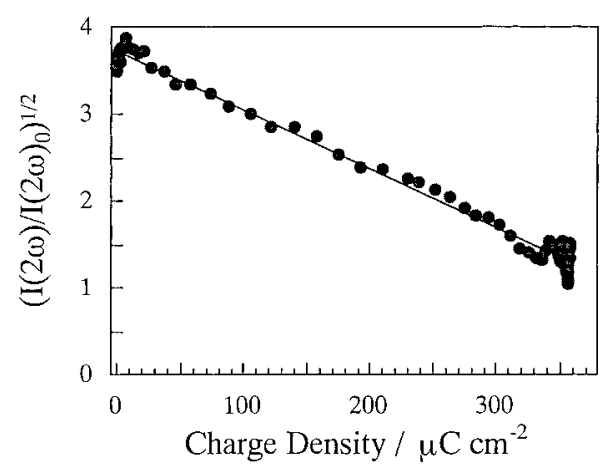

Figure 9. Plot of the square root of the normalized SHG signal (266 $\mathrm{nm}$ ) against the electric charge density for the $\mathrm{CO}$ oxidation using eq 6. The solid line shows a linear least squares fit. The excitation wavelength was $532 \mathrm{~nm}$.

oxidation process presented in Figure 4, where $\omega_{1}=\omega_{2}$. To calculate the charge density for CO oxidation, the double-layer charging current was subtracted assuming that the current in the double-layer region was independent of potential. The value of $\left(I(2 \omega) / I(2 \omega)_{0}\right)^{1 / 2}$ was calculated by using the SHG signal at $+0.2 \mathrm{~V}$ of the second scan in Figure $3 \mathrm{~b}$ as $I(2 \omega)_{0}$ with the assumption that $I(2 \omega)_{0}$ was independent of potential. The linear relation in Figure 9 shows that eq 6 is valid in this case and the change of the SHG signal reflected only the inherent resonance nature of the bonding between the surface and $\mathrm{CO}$, and the additional terms such as the adsorbate-adsorbate interaction are negligible.

\section{Conclusion}

The NLO responses of CO adsorbed on a Pt electrode surface were investigated with various excitation wavelengths. The NLO signal was enhanced by the adsorption of CO in all the cases, and the enhancement factor of the NLO signal by the adsorption of $\mathrm{CO}$ depended on the three-wave-mixing wavelength. Different enhancement mechanisms seemed to be applicable for visible and infrared excitation. At the infrared excitation, the SHG signal changed with potential, reflecting a change in the surface charge density. On the other hand, in the case of the visible excited SHG and near-infrared+visibleexcited SFG measurements, resonant NLO enhancement coupled with the electronic transition from the $E_{\mathrm{f}}$ of Pt to $2 \pi_{\mathrm{a}}{ }^{*}$ of CO(a) was observed. These results demonstrated the usefulness of NLO measurement, particularly with wavelength variability for in situ study of the molecular electronic structure at electrochemical interfaces.

Acknowledgment. This work was partially supported by Grants-in-Aid for Priority Area Research (06239204, 07228204, and 08218204) from the Ministry of Education, Science, Sports and Culture, Japan. I.Y. acknowledges the Japan Society for the Promotion of Science for the JSPS Research Fellowship for Young Scientists.

\section{References and Notes}

(1) Capton, A.; Parsons, R. J. Electroanal. Chem. 1973, 45, 205.

(2) Parsons, R.; VanderNoot, T. J. Electroanal. Chem. 1988, 257, 9.

(3) McNichol, B. D. J. Electroanal. Chem. 1981, 118, 71.

(4) Lamy, C. Electrochim. Acta 1984, 29, 1581.

(5) Sun, S. G.; Lin, Y.; Li, N. H.; Mu, J. Q. J. Electroanal. Chem. 1994, 370, 273.

(6) Sun, S. G.; Clavilier, J.; Bewick, A. J. Electroanal. Chem. 1988, $240,147$. 47.

(7) Beden, B.; Bewick, A.; Lamy, C. J. Electroanal. Chem. 1983, 148,

(8) Kunimatsu, K.; Kita, H. J. Electroanal. Chem. 1987, 218, 155.

(9) Chang, S. C.; Ho, Y. H.; Weaver, M. J. Surf. Sci. 1992, $265,81$. 
(10) Nishimura, K.; Ohnishi, R.; Kunimatsu, K.; Enyo, M. J. Electroanal. Chem. 1989, 258, 219.

(11) Iwashita, T.; Nart, F. C.; Lopez, B.; Vielstich, W. Electrochim. Acta 1992, 37, 2361.

(12) Zhang, Y.; Weaver, M. J. Langmuir 1993, 9, 1397.

(13) Ertl, G.; Neumann, M.; Streit, K. M. Surf. Sci. 1977, 64, 393

(14) Heskett, D.; Plummer, E. W.; Messmer, R. P. Surf. Sci. 1984, 139, 558 .

(15) Ferrer, S.; Frank, K. H.; Reihl, B. Surf. Sci. 1985, 162, 264.

(16) Dose, V. Surf. Sci. Rep. 1985, 5, 337.

(17) Rangelov, G.; Mammel, N.; Bertel, E.; Dose, V. Surf. Sci. 1991, 251/252, 965 .

(18) Wästberg, B.; Rosén, A. Surf. Sci. Lett. 1988, 193, L7.

(19) Kunimatsu, K.; Golden, W. G.; Seki, H.; Philpott, M. R. Langmuir $1985,1,245$.

(20) Kunimatsu, K.; Seki, H.; Golden, W. G.; Gordon, J. G., II; Philpott, M. R. Langmuir 1986, 2, 464.

(21) Lambert, D. K. Electrochim. Acta 1996, 41, 623.

(22) Holloway, S.; Norskov, J. K. J. Electroanal. Chem. 1984, 161, 193.

(23) Ikezawa, Y.; Fujisawa, H.; Ishii, F. Surf. Sci. 1989, 218, 246.

(24) Chang, S. C.; Weaver, M. J. J. Phys. Chem. 1990, 94, 5095.

(25) Chang, S. C.; Jiang, X.; Roth, J. D.; Weaver, M. J. J. Phys. Chem. 1991, 95, 5378 .

(26) Jiang, X.; Weaver, M. J. Surf. Sci. 1992, 275, 237.

(27) Roth, J. D.; Weaver, M. J. Langmuir 1992, 8, 1451.

(28) Shimazu, K.; Kaneda, K.; Kita, H. Bull. Chem. Soc. Jpn. 1994, 67, 2069.

(29) Inzelt, G.; Kertész, V.; Láng, G. J. Phys. Chem. 1993, 97, 6104.

(30) Inzelt, G.; Kertész, V. Electrochim. Acta 1993, 38, 2385.

(31) Zou, S.; Weaver, M. J. J. Phys. Chem. 1996, 100, 4237.

(32) Shen, Y. R. The Principles of Nonlinear Optics; Wiley: New York, 1984.

(33) Roy, D. Electrochim. Acta 1994, 39, 2699.

(34) Aktsipetrov, O. A.; Melnikov, A. V.; Murzina, T. V.; Nikulin, A. A.; Rubtsov, A. N. Surf. Sci. 1995, 336, 225.

(35) Guyot-Sionnest, P.; Tadjeddine, A. J. Chem. Phys. 1990, 92, 734.

(36) Fisher, P. R.; Daschbach, J. L.; Gragson, D. E.; Richmond, G. L.

J. Vac. Sci. Technol. A 1994, 12, 2617.

(37) Richmond, G. L. In Advances in Electrochemical Science, and Engineering; Gerischer, E., Tobias, C. W., Eds.; VCH: New York, 1991; Vol. 2.

(38) Liebsch, A. Surf. Sci. 1994, 307-309, 1007.

(39) Heinz, T. F. In Nonlinear Surface Electromagnetic Phenomena;

Ponath, H. E., Stegeman, G. I., Eds.; North-Holland: Amsterdam, 1991.

(40) Corn, R. M.; Higgins, D. A. Chem. Rev. 1994, 94, 107.

(41) Richmond, G. L.; Robinson, J. M.; Shannon, V. L. Prog. Surf. Sci.

1988, 28,1 .

(42) McGilp, J. F. Prog. Surf. Sci. 1995, 49, 1.

(43) Jordan, C.; Cant-Said, E. J.; Marowski, G. Appl. Phys. B 1994, 58, 111.

(44) Meyer, C.; Lüpke, G.; Emmerichs, U.; Wolter, F.; Kurz, H. Phys. Rev. Lett. 1995, 74, 3001.

(45) Bradley, R. A.; Georgiadis, R.; Kevan, S. D.; Richmond, G. L. J. Chem. Phys. 1993, 99, 5535.

(46) Gruzdkov, Y. A.; Shamardin, O. V.; Parmon, V. N. J. Chem. Soc., Faraday Trans. 1992, 88, 2705.

(47) Li, C. M.; Urbach, L. E.; Dai, H. L. Phys. Rev. B 1994, 49, 2104

(48) Lüpke, G.; Bottomley, D. J.; van Driel, H. M. Phys. Rev. B 1994, 49, 17303.

(49) Petrocelli, G.; Martellucci, S.; Francini, R. Appl. Phys. A 1993, $56,263$.
(50) Eisenthal, K. B. Annu. Rev. Phys. Chem. 1992, 43, 621.

(51) Naujok, R. R.; Higgins, D. A.; Hanken, D. G.; Corn, R. M. J. Chem. Soc., Faraday Trans. 1995, 91, 1411.

(52) Rasing, T.; Shen, Y. R. Phys. Rev. Lett. 1985, 55, 2903.

(53) Lynch, M. L.; Barner, B. J.; Lantz, J. M.; Corn, R. M. J. Chim. Phys. 1991, 88, 1271.

(54) Pettinger, B.; Lipkowski, J.; Mirwald, S.; Friedrich, A. J. Electroanal. Chem. 1992, 329, 289.

(55) Heinz, T. F.; Loy, M. M. T.; Thompson, W. A. Phys. Rev. Lett. 1985, 54, 63.

(56) Shen, Y. R. Nature 1989, 337, 519.

(57) Buck, M. Appl. Phys. A 1992, 55, 395.

(58) Bain, C. D. J. Chem. Soc., Faraday Trans. 1995, 91, 1281.

(59) Heinz, T. F.; Himpsel, F. J.; Palange, E. Phys. Rev. B. 1989, 63 , 644.

(60) Daum, W.; Keause, H.-J.; Reichel, U.; Ibach, H. Phys. Rev. Lett. 1993, $71,1234$.

(61) He, G.; Elking, M. D.; Xu, Z. Chem. Phys. Lett. 1996, 254, 184.

(62) Yagi, I.; Nakabayashi, S.; Uosaki, K. Chem. Lett. 1996, 529.

(63) Nakabayashi, S.; Sugiyama, N.; Yagi, I.; Uosaki, K. Chem. Phys.

1996, 205, 269

(64) Yagi, I.; Lantz, J. M.; Nakabayashi, S.; Corn, R. M.; Uosaki, K. J. Electroanal. Chem. 1996, 401, 95.

(65) Campbell, D. J.; Corn, R. M. J. Phys. Chem. 1988, 92, 5796.

(66) Lynch, M. L.; Barner, B. J.; Corn, R. M. J. Electroanal. Chem.

1991, 300, 447.

(67) Leger, J. M.; Beden, B.; Lamy, C. J. Electroanal. Chem. 1984, $170,305$.

(68) Beden, B.; Lamy, C.; deTacconi, N. R.; Arivia, A. J. Electrochim. Acta 1990, 35, 691.

(69) Grubb, S. G.; DeSantolo, A. M.; Hall, R. B. J. Phys. Chem. 1988, $92,1419$.

(70) Bae, I. T. J. Phys. Chem. 1996, 100, 14081.

(71) Guyot-Sionnest, P.; Chen, W.; Shen, Y. R. Phys. Rev. B 1986, 33, 8254.

(72) Bloembergen, N.; Chang, R. K.; Jha, S. S.; Lee, C. H. Phys. Rev. 1968, 174,813 .

(73) Mizrahi, V.; Sipe, J. E. J. Opt. Soc. Am. B. 1988, 5, 660.

(74) Richmond, G. L. Langmuir 1986, 2, 132.

(75) Orts, J. M.; Fernandez-Vega, A.; Feliu, J. M.; Aldaz, A.; Clavilier, J. J. Electroanal. Chem. 1992, 327, 261.

(76) Shen, Y. R. Surf. Sci. 1994, 299/300, 551.

(77) Collas, N.; Beden, B.; Leger, J. M.; Lamy, C. J. Electroanal. Chem. 1985, 186, 287

(78) Ikezawa, Y.; Nagashima, N.; Shibata, T.; Takamura, T. J. Electroanal. Chem. 1994, 365, 261.

(79) Caram, J. A.; Gutiérrez, C. J. Electroanal. Chem. 1990, 291, 289.

(80) Caram, J. A.; Gutiérrez, C. J. Electroanal. Chem. 1991, 305, 259.

(81) Costa, A.; Gutiérrez, C. J. Electroanal. Chem. 1995, 383, 195.

(82) Fukutani, K.; Song, M. B. Murata, Y. Faraday Discuss. 1993, 96, 105.

(83) Fukutani, K.; Song, M. B.; Murata, Y. J. Chem. Phys. 1995, 103, 2221

(84) Schmidt, M. E.; Guyot-Sionnest, P. J. Chem. Phys. 1996, 104, 2438.

(85) Nakatsuji, H.; Morita, H.; Nakai, H.; Murata, Y.; Fukutani, K. J. Chem. Phys. 1996, 104, 714

(86) Shelton, D. P.; Rice, J. E. Chem. Rev. 1994, 94, 3.

(87) Ward, J. F.; Miller, K. Phys. Rev. A 1979, 19, 826

(88) Wong, E. K. L.; Richmond, G. L. J. Chem. Phys. 1993, 99, 5500. 